\title{
Protein substrates engage the lumen of O-GIcNAc transferase's tetratricopeptide repeat domain in different ways
}

Cassandra M. Joiner[1]*, Forrest A. Hammel[1][2], John Janetzko[1][3], Suzanne Walker[1]*

Author Address

[1] Department of Microbiology, Harvard Medical School, 4 Blackfan Circle, Boston, MA 02115, USA

[2] Program in Chemical Biology, Harvard University, 12 Oxford Street, Cambridge, MA 02138, USA

[3] Department of Chemistry and Chemical Biology, Harvard University, 12 Oxford Street, Cambridge, MA 02138, USA

\author{
${ }^{*}$ Corresponding authors. \\ E-mails: Suzanne_walker@hms.harvard.edu \\ Joiner1@stolaf.edu
}

Supplemental Table S1-S7

Supplemental Figures S1-S6

General Methods

Tables of Plasmids

Tables of Primers

Table of Gene Blocks

Construction of pET24-8XHIS-HRV3C-ncOGT variants

Construction of TAB1 S395A and CAMKIV S189A variants

Bacterial expression and purification of pET24-8XHIS-HRV3C-ncOGT variants

In vitro glycosylation of HeLa extracts by ncOGT variants

Bacterial expression and purification of OGT substrates

In vitro glycosylation of OGT substrates by ncOGT variants

In vitro turnover assay of TAB1 glycosylation by ncOGT variants

In vitro time-dependent glycosylation of CAMKIV by ncOGT variants 
Supplemental Table S1. OGT Asparagine Ladder Conservation Table

\begin{tabular}{|c|c|c|}
\hline & \multicolumn{2}{|c|}{$\%$ Conservation per amino acid } \\
\hline Residue & $\mathbf{N}$ & Next highest cons. residues \\
\hline N458 & 88.9 & $2.7-$ S \\
\hline N424 & 96.3 & $1.0-\mathrm{S}, 1.0-\mathrm{A}$ \\
\hline N390 & 95.6 & $1.7-\mathrm{S}$ \\
\hline N356 & 95.9 & $1.7-\mathrm{S}$ \\
\hline N322 & 95.9 & $1.7-\mathrm{D}$ \\
\hline N288 & 94.2 & $1.7-\mathrm{S}, 1.7-\mathrm{K}$ \\
\hline $\mathrm{N} 254$ & 91.8 & $3.2-\mathrm{S}$ \\
\hline $\mathrm{N} 220$ & 90.7 & $3.0-\mathrm{A}$ \\
\hline $\mathrm{N} 186$ & 93.1 & $4.1-\mathrm{D}$ \\
\hline $\mathrm{N} 118$ & 82.7 & $7.6-\mathrm{L}$ \\
\hline $\mathrm{N} 84$ & 83.6 & $3.6-\mathrm{S}$ \\
\hline
\end{tabular}

Conservation scores determined for OGT's TPR domain using the 1W3B PDB file and ConSurf Server. ${ }^{1-3} 150$ sequence homologs were compared.

\section{Supplemental Table S2. OGT Aspartate Ladder Conservation Table}

\begin{tabular}{|c|c|c|}
\hline & \multicolumn{2}{|c|}{ \% Conservation per amino acid } \\
\hline Residue & D & Next highest cons. residues \\
\hline D454 & 54.5 & $33.8-\mathrm{E}$ \\
\hline D420 & 53.2 & $38.1-\mathrm{E}$ \\
\hline D386 & 81.8 & $9.8-\mathrm{E}$ \\
\hline D318 & 49.5 & $11.9-\mathrm{E}$ \\
\hline D284 & 55.9 & $34.8-\mathrm{E}$ \\
\hline D216 & 80.6 & $13.6-\mathrm{E}$ \\
\hline D152 $^{*}$ & 45.9 & $48.1-\mathrm{N}$ \\
\hline D114 & 85.8 & $4.4-\mathrm{E}$ \\
\hline
\end{tabular}

* Position 152 can be either an aspartate (D - 45.9\% conservation) or asparagine ( $\mathrm{N}-48.1 \%$ conservation). Conservation scores determined for OGT's TPR domain using the 1W3B PDB file and ConSurf Server. ${ }^{1-3} 150$ sequence homologs were compared. 
Supplemental Table S3. Quantification of in vitro glycosylation of purified substrates by OGT TPR truncation mutants

\begin{tabular}{|c|c|c|c|c|c|c|c|c|c|c|c|c|}
\hline \multirow[t]{2}{*}{$\begin{array}{l}\text { OGT } \\
\text { constructs }\end{array}$} & \multicolumn{3}{|c|}{$\begin{array}{l}\text { TAB1 (1-504) } \\
\text { Glycosylation Signal } \\
\text { (Fold change } \\
\text { compared to OGT WT) }\end{array}$} & \multicolumn{3}{|c|}{$\begin{array}{c}\text { TAB1 (1-402) } \\
\text { Glycosylation Signal } \\
\text { (Fold change } \\
\text { compared to OGT } \\
\text { WT) }\end{array}$} & \multicolumn{3}{|c|}{$\begin{array}{c}\text { CARM1 } \\
\text { Glycosylation Signal } \\
\text { (Fold change } \\
\text { compared to OGT } \\
\text { WT) }\end{array}$} & \multicolumn{3}{|c|}{$\begin{array}{c}\text { CAMKIV } \\
\text { Glycosylation Signal } \\
\text { (Fold change } \\
\text { compared to OGT } \\
\text { WT) }\end{array}$} \\
\hline & $\operatorname{Rep} 1$ & $\operatorname{Rep} 2$ & Avg & $\operatorname{Rep} 1$ & $\operatorname{Rep} 2$ & Avg & $\operatorname{Rep} 1$ & $\operatorname{Rep} 2$ & Avg & $\operatorname{Rep} 1$ & $\operatorname{Rep} 2$ & Avg \\
\hline OGT WT & 1.0 & 1.0 & 1.00 & 1.0 & 1.0 & 1.00 & 1.0 & 1.0 & 1.00 & 1.0 & 1.0 & 1.00 \\
\hline OGT $\Delta 1$ & 0.1 & 0.1 & 0.10 & 1.1 & 1.0 & 1.05 & 0.8 & 0.8 & 0.80 & 0.2 & 0.5 & 0.35 \\
\hline OGT $\Delta 2$ & 0.0 & 0.1 & 0.05 & 1.0 & 1.0 & 1.00 & 0.7 & 0.7 & 0.70 & 0.5 & 0.5 & 0.50 \\
\hline OGT $\Delta 3$ & 0.0 & 0.1 & 0.05 & 1.4 & 1.0 & 1.20 & 0.7 & 0.8 & 0.75 & 1.7 & 1.2 & 1.45 \\
\hline OGT $\Delta 4$ & 0.1 & 0.2 & 0.15 & 1.3 & 1.0 & 1.15 & 0.7 & 0.9 & 0.80 & 2.4 & 2.0 & 2.20 \\
\hline OGT $\Delta 5$ & 0.1 & 0.4 & 0.25 & 1.4 & 1.1 & 1.25 & 0.8 & 1.0 & 0.90 & 2.8 & 2.5 & 2.65 \\
\hline OGT $\Delta 6$ & 0.1 & 0.3 & 0.20 & 1.5 & 1.0 & 1.25 & 0.9 & 1.0 & 0.95 & 2.0 & 1.8 & 1.90 \\
\hline
\end{tabular}

Supplemental Table S4. Quantification of in vitro glycosylation of purified substrates by OGT N5A mutants

\begin{tabular}{|c|c|c|c|c|c|c|c|c|c|c|c|c|}
\hline \multirow[t]{2}{*}{$\begin{array}{l}\text { OGT } \\
\text { constructs }\end{array}$} & \multicolumn{3}{|c|}{$\begin{array}{c}\text { TAB1 (1-504) } \\
\text { Glycosylation Signal } \\
\text { (Fold change } \\
\text { compared to OGT WT) }\end{array}$} & \multicolumn{3}{|c|}{$\begin{array}{c}\text { TAB1 (1-402) } \\
\text { Glycosylation Signal } \\
\text { (Fold change } \\
\text { compared to OGT } \\
\text { WT) }\end{array}$} & \multicolumn{3}{|c|}{$\begin{array}{c}\text { CARM1 } \\
\text { Glycosylation Signal } \\
\text { (Fold change } \\
\text { compared to OGT } \\
\text { WT) }\end{array}$} & \multicolumn{3}{|c|}{$\begin{array}{c}\text { CAMKIV } \\
\text { Glycosylation Signal } \\
\text { (Fold change } \\
\text { compared to OGT } \\
\text { WT) }\end{array}$} \\
\hline & $\operatorname{Rep} 1$ & $\operatorname{Rep} 2$ & Avg & $\operatorname{Rep} 1$ & $\operatorname{Rep} 2$ & Avg & $\operatorname{Rep} 1$ & $\operatorname{Rep} 2$ & Avg & $\operatorname{Rep} 1$ & $\operatorname{Rep} 2$ & Avg \\
\hline OGT WT & 1.0 & 1.0 & 1.00 & 1.0 & 1.0 & 1.00 & 1.0 & 1.0 & 1.00 & 1.0 & 1.0 & 1.00 \\
\hline OGT N5Aprox & 0.1 & 0.1 & 0.10 & 1.1 & 1.0 & 1.05 & 1.0 & 1.0 & 1.00 & 0.4 & 0.4 & 0.40 \\
\hline OGT N5Amed & 0.1 & 0.1 & 0.10 & 1.1 & 1.0 & 1.05 & 1.0 & 1.0 & 1.00 & 0.5 & 0.5 & 0.50 \\
\hline OGT N5Adist & 0.2 & 0.2 & 0.20 & 1.1 & 0.9 & 1.00 & 0.9 & 0.9 & 0.90 & 1.1 & 1.1 & 1.10 \\
\hline
\end{tabular}

Supplemental Table S5. Quantification of in vitro glycosylation of purified substrates by OGT D2A mutants

\begin{tabular}{|c|c|c|c|c|c|c|c|c|c|c|c|c|}
\hline \multirow[t]{2}{*}{$\begin{array}{l}\text { OGT } \\
\text { constructs }\end{array}$} & \multicolumn{3}{|c|}{$\begin{array}{c}\text { TAB1 (1-504) } \\
\text { Glycosylation Signal } \\
\text { (Fold change } \\
\text { compared to OGT WT) }\end{array}$} & \multicolumn{3}{|c|}{$\begin{array}{c}\text { TAB1 (1-402) } \\
\text { Glycosylation Signal } \\
\text { (Fold change } \\
\text { compared to OGT } \\
\text { WT) }\end{array}$} & \multicolumn{3}{|c|}{$\begin{array}{c}\text { CARM1 } \\
\text { Glycosylation Signal } \\
\text { (Fold change } \\
\text { compared to OGT WT) }\end{array}$} & \multicolumn{3}{|c|}{$\begin{array}{c}\text { CAMKIV } \\
\text { Glycosylation Signal } \\
\text { (Fold change } \\
\text { compared to OGT } \\
\text { WT) }\end{array}$} \\
\hline & $\operatorname{Rep} 1$ & $\operatorname{Rep} 2$ & Avg & $\operatorname{Rep} 1$ & $\operatorname{Rep} 2$ & Avg & $\operatorname{Rep} 1$ & $\operatorname{Rep} 2$ & Avg & $\operatorname{Rep} 1$ & $\operatorname{Rep} 2$ & Avg \\
\hline OGT WT & 1.0 & 1.0 & 1.00 & 1.0 & 1.0 & 1.00 & 1.0 & 1.0 & 1.00 & 1.0 & 1.0 & 1.00 \\
\hline $\begin{array}{c}\text { OGT D2A- } \\
1_{\text {prox }}\end{array}$ & 2.2 & 2.4 & 2.30 & 1.2 & 0.9 & 1.05 & 0.9 & 1.0 & 0.95 & 1.8 & 1.7 & 1.75 \\
\hline $\begin{array}{c}\text { OGT D2A- } \\
2 \text { prox }\end{array}$ & 2.2 & 2.5 & 2.35 & 1.1 & 0.9 & 1.00 & 1.4 & 1.3 & 1.35 & 2.7 & 2.9 & 2.80 \\
\hline $\begin{array}{c}\text { OGT D2A- } \\
\text { 3med }\end{array}$ & 2.0 & 2.3 & 2.15 & 1.1 & 1.0 & 1.05 & 1.3 & 1.5 & 1.40 & 8.1 & 7.8 & 7.95 \\
\hline $\begin{array}{c}\text { OGT D2A- } \\
\text { 4dist }^{\text {Ond }}\end{array}$ & 0.9 & 0.9 & 0.90 & 1.1 & 1.0 & 1.05 & 1.2 & 1.3 & 1.25 & 3.1 & 3.1 & 3.10 \\
\hline $\begin{array}{c}\text { OGT D2A- } \\
\text { 5dist }\end{array}$ & 0.8 & 0.7 & 0.75 & 1.1 & 1.0 & 1.05 & 1.6 & 1.4 & 1.50 & 3.1 & 3.1 & 3.10 \\
\hline
\end{tabular}


Supplemental Table S6. Quantification of in vitro turnover of TAB1 glycosylation by OGT WT and D2A2 prox

\begin{tabular}{|c|c|c|c|c|}
\hline Time (min) & $\begin{array}{c}\text { OGT WT_Rep 1 } \\
\text { (densitometry) }\end{array}$ & $\begin{array}{c}\text { OGT WT_Rep 2 } \\
\text { (densitometry) }\end{array}$ & $\begin{array}{c}\text { OGT D2A- } \\
\text { 2prox_Rep1 } \\
\text { (densitometry) }\end{array}$ & $\begin{array}{c}\text { OGT D2A- } \\
\text { 2prox_Rep2 } \\
\text { (densitometry) }\end{array}$ \\
\hline 0.0 & 2.2 & 4.5 & 2.3 & 2.2 \\
\hline 1.0 & 7.5 & 5.9 & 43.8 & 37.7 \\
\hline 2.5 & 16.1 & 10.4 & 68.9 & 62.9 \\
\hline 5.0 & 25.0 & 20.8 & 86.3 & 84.1 \\
\hline 10.0 & 41.0 & 32.9 & 92.7 & 97.7 \\
\hline 20.0 & 60.1 & 48.0 & 95.0 & 104.3 \\
\hline 30.0 & 63.0 & 55.5 & 97.1 & 107.0 \\
\hline
\end{tabular}

Supplemental Table S7. Quantification of in vitro turnover of TAB1 S395A glycosylation by OGT WT and D2A-2 prox

\begin{tabular}{|c|c|c|c|c|}
\hline Time (min) & $\begin{array}{c}\text { OGT WT_Rep 1 } \\
\text { (densitometry) }\end{array}$ & $\begin{array}{c}\text { OGT WT_Rep 2 } \\
\text { (densitometry) }\end{array}$ & $\begin{array}{c}\text { OGT D2A- } \\
\text { 2prox_Rep1 } \\
\text { (densitometry) }\end{array}$ & $\begin{array}{c}\text { OGT D2A- } \\
\text { 2prox_Rep2 } \\
\text { (densitometry) }\end{array}$ \\
\hline 0.0 & 0.8 & 1.2 & 0.0 & 0.0 \\
\hline 1.0 & 0.8 & 1.2 & 0.0 & 0.1 \\
\hline 5.0 & 0.8 & 1.6 & 2.3 & 2.0 \\
\hline 10.0 & 0.9 & 2.0 & 7.9 & 4.0 \\
\hline 20.0 & 1.8 & 1.8 & 6.7 & 5.9 \\
\hline 30.0 & 2.0 & 1.2 & 7.1 & 6.8 \\
\hline
\end{tabular}


A TPR Domain
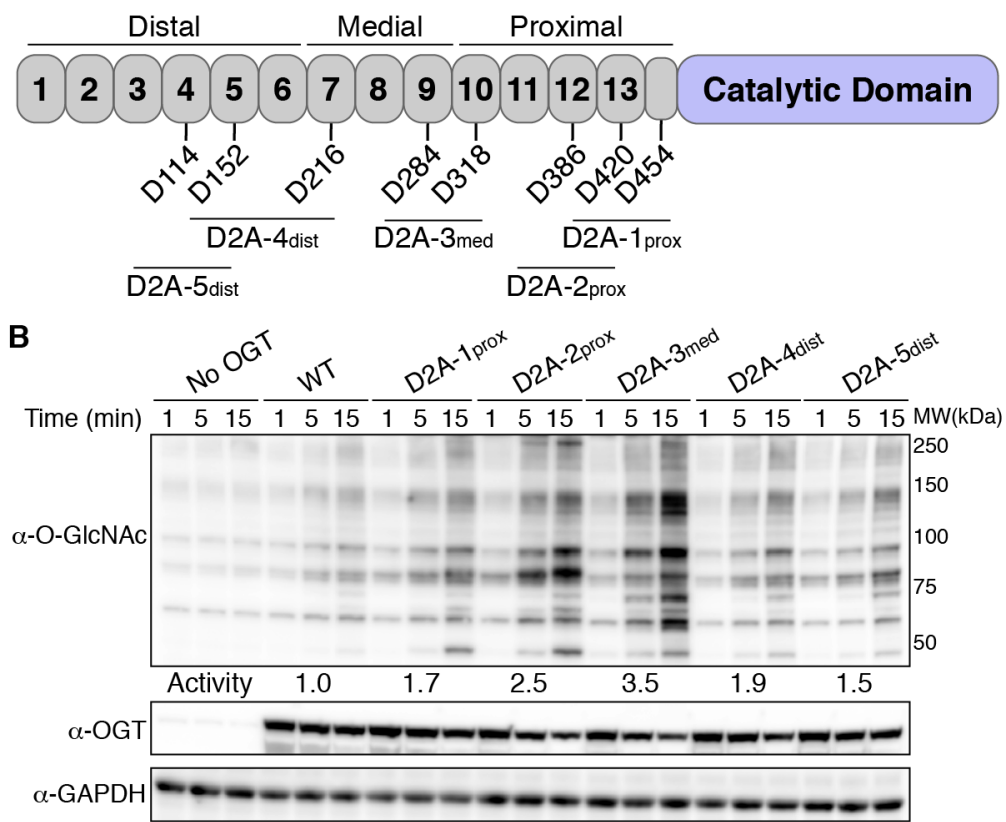

Supplemental Figure S1. Aspartate ladder differentially regulates global glycosylation of HeLa extracts. (A) Cartoon schematic of OGT D2A mutant constructs. (B) Glycosylation of HeLa extracts shows differential increases in glycosylation for D2A mutants with largest increases for D2A-2 and D2A-3. Representative blot for two independent experiments. See methods for relative glycosylation activity calculations. 
A
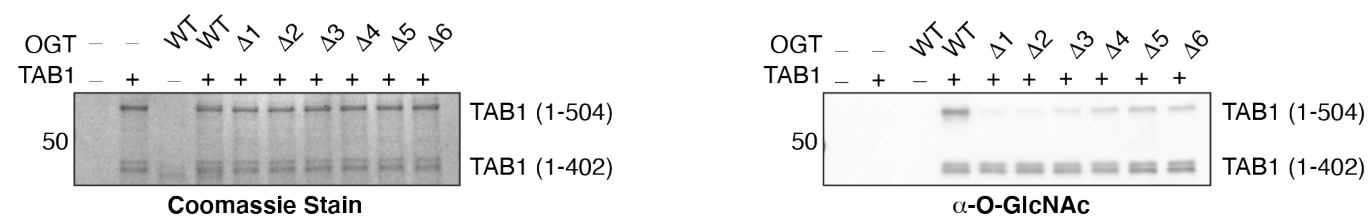

B
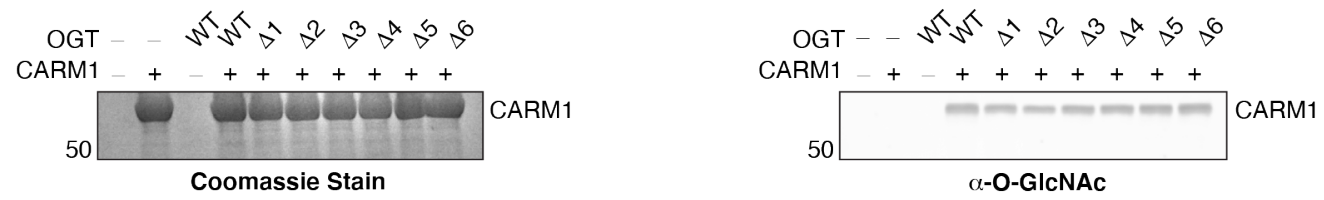

C
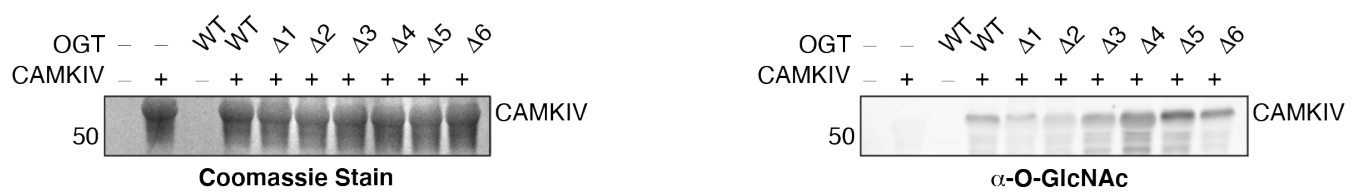

Supplemental Figure S2. SDS-PAGE gels and western blots of in vitro glycosylation of OGT substrates by OGT TPR truncation variants. A) In vitro glycosylation of TAB1 by TPR truncation mutants. B) In vitro glycosylation of CARM1 by TPR truncation mutants. C) In vitro glycosylation of CAMKIV by TPR truncation mutants. Glycosylation assays were run for 90 minutes. All SDS-PAGE gels were stained with Instant Blue Coomassie stain and western blots were visualized using the pan O-GICNAc antibody CTD110.6. Representative gels and blots are shown for two independent experiments for each data set. 
A
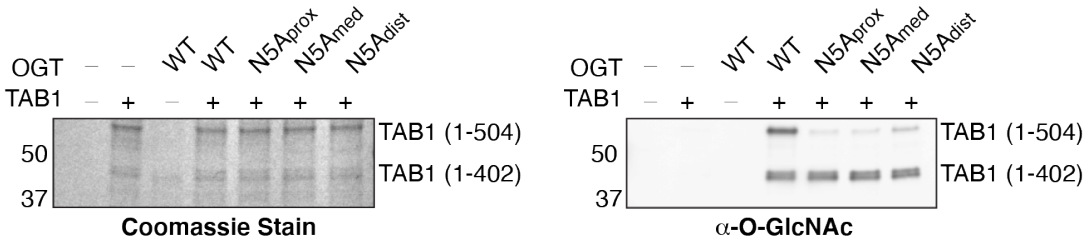

B
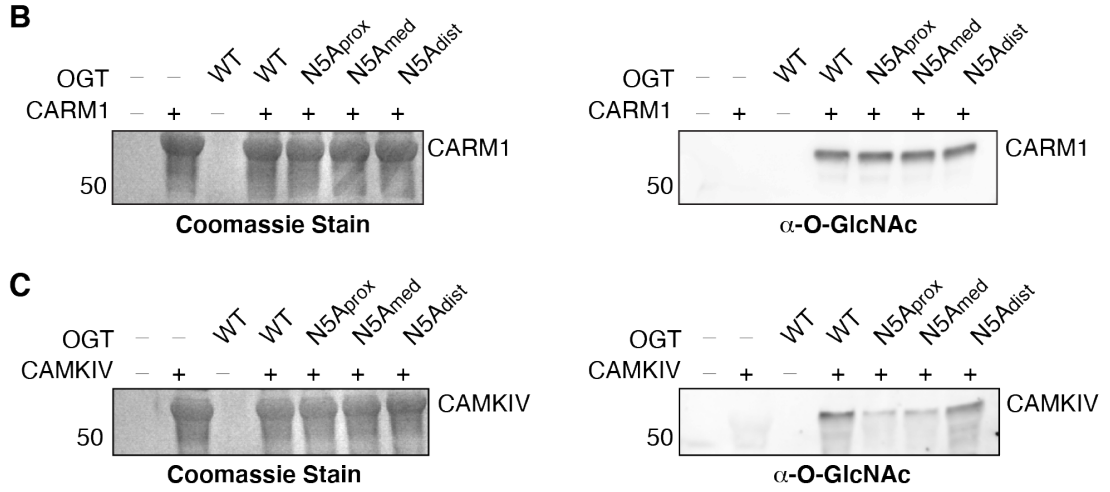

Supplemental Figure S3. SDS-PAGE gels and western blots of in vitro glycosylation of OGT substrates by OGT asparagine ladder variants. A) In vitro glycosylation of TAB1 by N5A mutants. B) In vitro glycosylation of CARM1 by N5A mutants. C) In vitro glycosylation of CAMKIV by N5A mutants. Glycosylation assays were run for 90 minutes. All SDS-PAGE gels were stained with Instant Blue Coomassie stain and western blots were visualized using the pan O-GIcNAc antibody CTD110.6. Representative gels and blots are shown for two independent experiments for each data set. 
A
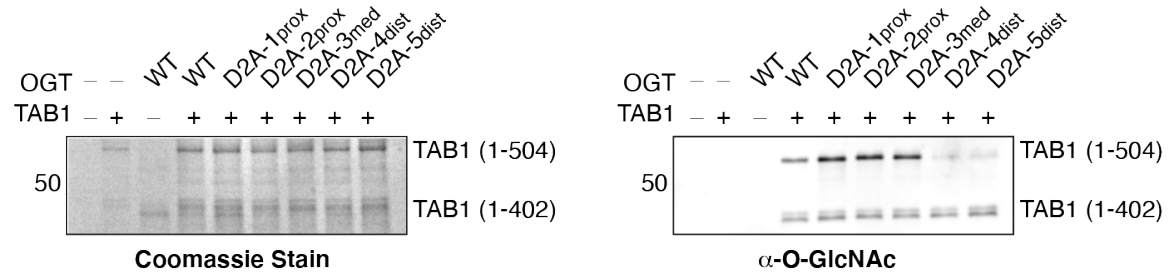

B
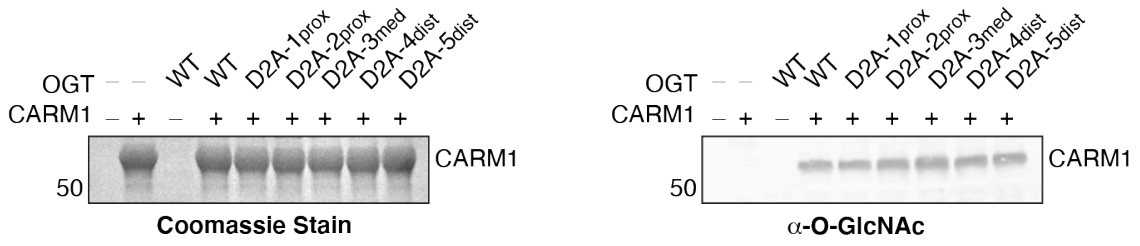

C
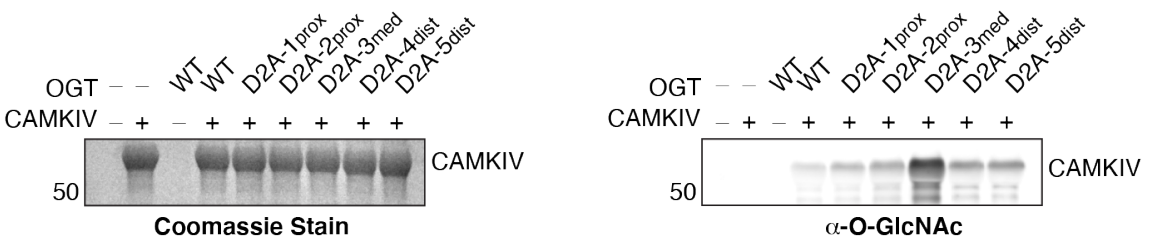

Supplemental Figure S4. Full length SDS-PAGE gels and western blots of in vitro glycosylation of OGT substrates by OGT aspartate ladder variants. A) In vitro glycosylation of TAB1 by D2A mutants. B) In vitro glycosylation of CARM1 by D2A mutants. C) In vitro glycosylation of CAMKIV by D2A mutants. Glycosylation assays were run for 90 minutes. All SDS-PAGE gels were stained with Instant Blue Coomassie stain and western blots were visualized using the pan O-GICNAc antibody CTD110.6. Representative gels and blots are shown for two independent experiments for each data set. 

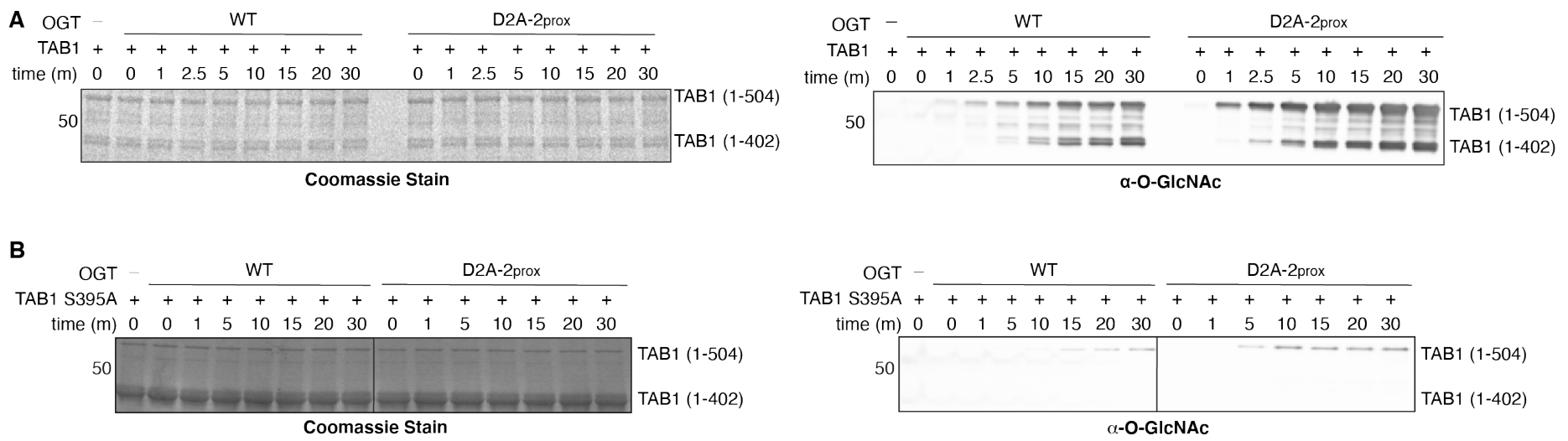

Supplemental Figure S5. SDS-PAGE gels and western blots of in vitro glycosylation of TAB1 WT and S395A by OGT variants. Time-dependent in vitro glycosylation of A) TAB1 WT and B) TAB1 S395A by OGT WT and D2A-2 2 rox. Western blot and gel images in panel $A$ and $B$ were acquired under identical conditions. Representative gels and blots are shown for two independent experiments. 
A

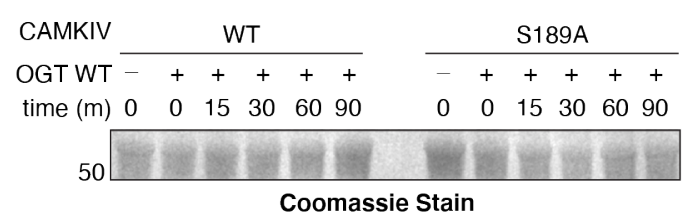

B

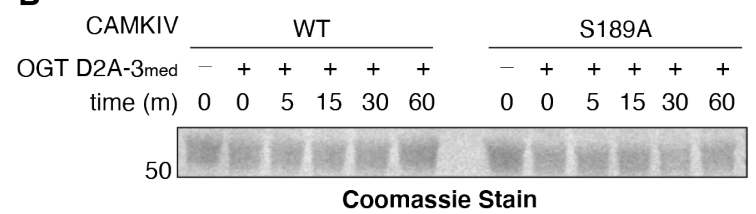

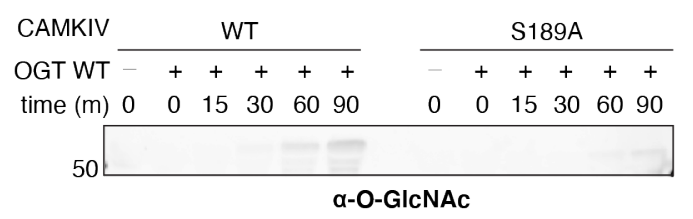

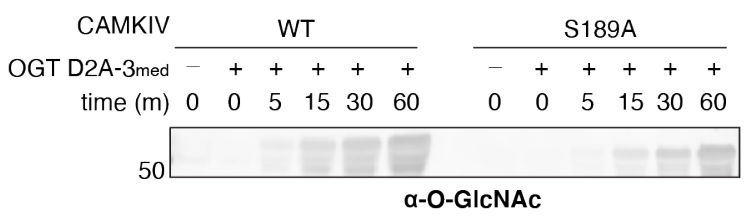

Supplemental Figure S6. Time dependent in vitro glycosylation of CAMKIV WT and S189A by OGT variants. A) Time-dependent in vitro glycosylation of CAMKIV WT and S189A by OGT WT shows that S189 is the major glycosite for CAMKIV. Max glycosylation of CAMKIV WT by wildtype OGT is seen at $90 \mathrm{~min}$. B) Timedependent in vitro glycosylation of CAMKIV WT and S189A by OGT D2A-3med shows glycosylation of CAMKIV WT as early as 5 minutes. D2A- $3_{\text {med }}$ shows increased glycosylation of CAMKIV compared to wildtype OGT. Western blots in panels $A$ and $B$ were imaged at the same time under identical conditions. Representative blots are shown for two independent experiments. 


\section{General Methods}

All primers and gene blocks were purchased from Integrated DNA Technologies (IDT) and all isolated plasmid were verified by sequencing by the Dana-Farber/Harvard Cancer Center DNA Resource Core (Boston, MA).

Table of Plasmids

\begin{tabular}{|c|c|c|}
\hline Plasmids & Function & Reference \\
\hline pET24b-8XHIS-HRV3C-ncOGT WT & Expresses 8XHIS-HRV3C-ncOGT (1-1036) & 6 \\
\hline pET24b-8XHIS-HRV3C-ncOGT N5A prox & $\begin{array}{l}\text { Expresses 8XHIS-HRV3C-ncOGT (1-1036) with } \\
\text { GCA codon replacing N322, N356, N390, N424, } \\
\text { and N458 }\end{array}$ & 7,8 \\
\hline pET24b-8XHIS-HRV3C-ncOGT N5A & $\begin{array}{l}\text { Expresses 8XHIS-HRV3C-ncOGT (1-1036) with } \\
\text { GCA codon replacing N186, N220, N254, N288, } \\
\text { and N322 }\end{array}$ & This study \\
\hline pET24b-8XHIS-HRV3C-ncOGT N5A dist & $\begin{array}{l}\text { Expresses 8XHIS-HRV3C-ncOGT (1-1036) with } \\
\text { GCA codon replacing N84, N118, N155, N186, } \\
\text { and N220 }\end{array}$ & This study \\
\hline $\begin{array}{l}\text { pET24b-8XHIS-HRV3C-ncOGT } \\
\text { D420A/D454A (D2A-1 } \text { prox }\end{array}$ & $\begin{array}{l}\text { Expresses 8XHIS-HRV3C-ncOGT }(1-1036) \text { with } \\
\text { GCA codon replacing the existing amino acids }\end{array}$ & 9 \\
\hline $\begin{array}{l}\text { pET24b-8XHIS-HRV3C-ncOGT } \\
\text { D386A/D420A (D2A-2 prox) }\end{array}$ & $\begin{array}{l}\text { Expresses 8XHIS-HRV3C-ncOGT }(1-1036) \text { with } \\
\text { GCA codon replacing the existing amino acids }\end{array}$ & 9 \\
\hline $\begin{array}{l}\text { pET24b-8XHIS-HRV3C-ncOGT } \\
\left.\text { D284A/D318A (D2A-3 }{ }_{\text {med }}\right)\end{array}$ & $\begin{array}{l}\text { Expresses 8XHIS-HRV3C-ncOGT (1-1036) with } \\
\text { GCA codon replacing the existing amino acids }\end{array}$ & This study \\
\hline $\begin{array}{l}\text { pET24b-8XHIS-HRV3C-ncOGT } \\
\text { D152A/D216A (D2A-4 dist) }\end{array}$ & $\begin{array}{l}\text { Expresses 8XHIS-HRV3C-ncOGT (1-1036) with } \\
\text { GCA codon replacing the existing amino acids }\end{array}$ & This study \\
\hline $\begin{array}{l}\text { pET24b-8XHIS-HRV3C-ncOGT } \\
\text { D114A/D152A (D2A-5 dist) }\end{array}$ & $\begin{array}{l}\text { Expresses 8XHIS-HRV3C-ncOGT }(1-1036) \text { with } \\
\text { GCA codon replacing the existing amino acids }\end{array}$ & This study \\
\hline $\begin{array}{l}\text { pET24b-8XHIS-HRV3C-ncOGT } \\
\text { D284A/D386A/D420A/D454A (D4A) }\end{array}$ & $\begin{array}{l}\text { Expresses 8XHIS-HRV3C-ncOGT (1-1036) with } \\
\text { GCA codon replacing the existing amino acids }\end{array}$ & This study \\
\hline pET24b-8XHIS-HRV3C-ncOGT $\Delta 1$ & $\begin{array}{l}\text { Expresses 8XHIS-HRV3C-ncOGT (323-1036) } \\
\text { containing 4.5 TPRs (TPRs 10-13.5) }\end{array}$ & 5 \\
\hline pET24b-8XHIS-HRV3C-ncOGT $\Delta 2$ & $\begin{array}{l}\text { Expresses 8XHIS-HRV3C-ncOGT (289-1036) } \\
\text { containing 5.5 TPRs (TPRs 9-13.5) }\end{array}$ & This study \\
\hline pET24b-8XHIS-HRV3C-ncOGT $\Delta 3$ & $\begin{array}{l}\text { Expresses 8XHIS-HRV3C-ncOGT (238-1036) } \\
\text { containing 6.5 TPRs (TPRs 8-13.5) }\end{array}$ & This study \\
\hline pET24b-8XHIS-HRV3C-ncOGT $\Delta 4$ & $\begin{array}{l}\text { Expresses } 8 \text { XHIS-HRV3C-ncOGT (221-1036) } \\
\text { containing 7.5 TPRs (TPRs 7-13.5) }\end{array}$ & This study \\
\hline pET24b-8XHIS-HRV3C-ncOGT $\Delta 5$ & $\begin{array}{l}\text { Expresses 8XHIS-HRV3C-ncOGT (203-1036) } \\
\text { containing 8.0 TPRs (TPRs 6.5-13.5) }\end{array}$ & This study \\
\hline pET24b-8XHIS-HRV3C-ncOGT $\Delta 6$ & $\begin{array}{l}\text { Expresses } 8 \text { XHIS-HRV3C-ncOGT (169-1036) } \\
\text { containing 9.0 TPRs (TPRs 5.5-13.5) }\end{array}$ & This study \\
\hline pET28a-CARM1 & Expresses polyhistidine-tagged CARM1 & 4 \\
\hline pET28a-CAMKIV & Expresses polyhistidine-tagged CAMKIV & 4 \\
\hline pET28a-TAB1 & Expresses polyhistidine-tagged TAB1 & 4 \\
\hline pET28a-TAB1 S395A & $\begin{array}{l}\text { Expresses polyhistidine-tagged TAB1 with GCA } \\
\text { codon replacing S395. }\end{array}$ & This study \\
\hline pET28a-CAMKIV S189A & $\begin{array}{l}\text { Expresses polyhistidine-tagged CAMKIV with } \\
\text { GCA codon replacing S189 }\end{array}$ & This study \\
\hline
\end{tabular}

Table of Primers

\begin{tabular}{|l|l|}
\hline \multicolumn{1}{|c|}{ Primer ID } & \multicolumn{1}{c|}{ Sequence } \\
\hline ncOGT N5A-2 FOR & 5' - GCAGACCAAGCAACAGCGAAGTTCGGCTGGGTTTCG - 3' \\
\hline ncOGT N5A-2 REV & 5' - GGCTAACATCAAACGTGAACAGGGTAACATCGAAGAAGCTG - 3' \\
\hline ncOGT N5A-3 FOR & 5' - GAGTAAGCTTCAGCCAGCAGCGGGTTCTGTTTGATAGC - 3' \\
\hline ncOGT N5A-3 REV & 5' - CACTGGGTAACGTTCTGAAAGAAGCTCGTATCTTCGACCGTGC - 3' \\
\hline
\end{tabular}




\begin{tabular}{|c|c|}
\hline ncOGT D318A FOR & 5' - GTGCCCGACCCACGCTGCATCTCTGAACAACCTGG - 3' \\
\hline ncOGT D318A REV & 5' - CCAGGTTGTTCAGAGATGCAGCGTGGGTCGGGCAC - 3' \\
\hline ncOGT D284A FOR & 5' - CAGCCGCACTTTCCAGCAGCTTACTGCAACCTGG - 3' \\
\hline ncOGT D284A REV & 5' - CCAGGTTGCAGTAAGCTGCTGGAAAGTGCGGCTG - 3' \\
\hline ncOGT D216A FOR & 5' - GGACCCGAACTTCCTGGCAGCTTACATCAACCTGG - 3' \\
\hline ncOGT D216A REV & 5' - CCAGGTTGATGTAAGCTGCCAGGAAGTTCGGGTCC - 3' \\
\hline ncOGT D152A FOR & 5' - CGTTCGTTCTGCACTGGGTAACCTG - 3' \\
\hline ncOGT D152A REV & 5' - CAGTACAGGTCCGGGTTG - 3' \\
\hline ncOGT D114A FOR & 5' - GAAAACGGACTTCATCGCAGGTTACATCAACCTGGCTG - 3' \\
\hline ncOGT D114A REV & 5' - CAGCCAGGTTGATGTAACCTGCGATGAAGTCCGGTTTC - 3' \\
\hline TAB1 S395A FOR & 5' - GTGTCTGTGCCATACGCAAGCGCCCAGAGCACC - 3' \\
\hline TAB1 S395A REV & 5' - GGTGCTCTGGGCGCTTGCGTATGGCACAGACAC - 3' \\
\hline CAMKIV S189A FOR & 5' - CCAAATCGCTGATTTTGGACTCGCAAAAATTGTGGAACATCAAGTGCTC - 3' \\
\hline CAMKIV S189A REV & 5' - GAGCACTTGATGTTCCACAATTTTTGCGAGTCCAAAATCAGCGATTTTG - 3' \\
\hline
\end{tabular}

Table of Gene Blocks

\begin{tabular}{|l|l|}
\hline \multicolumn{1}{|c|}{ Gene Block ID } & \multicolumn{1}{c|}{ Sequence } \\
\hline ncOGT N5A-2 & 5'- CGCTGTTGCTTGGTCTGCACTGGGTTGCGTTTTCAACGCTCAGGGTGAA \\
(N186A/N220A/N254A/N288A & ATCTGGCTGGCTATCCACCACTTCGAAAAGCTGTTACCCTGGACCCGAACT \\
/N322A) gene block & TCCTGGACGCTTACATCGCACTGGGTAACGTTCTGAAAGAAGCTCGTCGTAT \\
& CTTCGACCGTGCTGTTGCTGCTTACCTGCGTGCTCTGTCTCTGTCTCCGAAC \\
& CACGCTGTTGTTCACGGTGCACTGGCTTGCGTTTACTACGAACAGGGTCTGA \\
& TCGACCTGGCTATCGACACCTACCGTCGTGCTATCGAACTGCAGCCGCACTT \\
& TCCAGATGCTTACTGCGCACTGGCTAACGCTCTGAAAGAAAAGGTTCTGTT \\
& GCTGAAGCTGAAGACTGCTACAACACCGCTCTGCGTCTGTGCCCGACCCAC \\
\hline ncOGT N5A-3 & GCTGACTCTCTGAACGCACTGGCTAACATCAAACGTGAAC - 3' \\
(N84A/N118A/N155A/N186A/ & 5'- GCTGGCTGAAGCTTACTCTGCACTGGGTAACGTTTACAAGAACG \\
gene block & TGGTCAGCTGCAGGAAGCTATCGAACACTACCGTCACGCTCGCGTCT \\
& GAAACCGGACTTCATCGACGGTTACATCGCACTGGCTGCTGCTCTGG \\
& TTGCTGCTGGTGACATGGAAGGTGCTGTTCAGGCTTACGTTTCTGCTC \\
& TGCAGTACAACCCGGACCTGTACTGCGTTCGTTCTGACCTGGGTGCA \\
& CTGCTGAAAGCTCTGGGTCGTCTGGAAGAAGCTAAAGCTTGCTACCT \\
& GAAAGCTATCGAAACCCAGCCGAACTTCGCTGTTGCTTGGTCTGCAC \\
& TGGGTTGCGTTTTCAACGCTCAGGGTGAAATCTGGCTGGCTATCCAC \\
& CACTTCGAAAAAGCTGTTACCCTGGACCCGAACTTCCTGGACGCTTAC \\
\hline
\end{tabular}

\section{Construction of pet24-8XHIS-HRV3C-ncOGT variants}

All plasmids containing mutations within ncOGT's TPR domain were derived from the pET24b-8XHIS-HRV3CncOGT WT parent plasmid using the following methods:

Asparagine ladder variants: Using Gibson Assembly cloning, five sequential asparagine to alanine point mutations were made by inserting a $\sim 450$ base pair gene blocks containing the OGT sequences with the specific five sequence asparagine residues mutated to the GCA (alanine) codon. These gene blocks were designed to contain 15-20 base pair of homology on either end to the site of incorporation in the pET24b-8XHIS-HRV3CncOGT WT plasmid. Primers were designed to linearize the pET24b-8XHIS-HRV3C-ncOGT WT plasmid at either side of the given gene block insertion. Each set of primers for a given N5A variant were used to amplify linearized version of the parent plasmid without the region containing the mutations. The amplified PCR products were gel extracted and incubated with the Gibson Assembly Master Mix (NEB) at $50{ }^{\circ} \mathrm{C}$ for 1 hour. The assembled plasmids were transformed into STELLAR cloning cells and confirmed by Sanger sequencing.

Aspartate ladder and TPR truncation variants: Using QuickChange site-directed mutagenesis, single point mutations or truncation mutants were made by replacing the existing amino acid in the TPR lumen with the GCA (alanine) codon or deleting specific regions from the TPR domain. PCR primers were designed to have $\sim 15-20$ base pairs of homology on either side of the mutation or deletion site. Each set of primers for a given variant 
were used to amplify the TPR mutation using the parent plasmid as the template. The amplified PCR products were digested with the Dpn1 restriction enzyme to digest the parent plasmid, leaving the desired mutant plasmids. The digested DNA products were transformed into STELLAR or Nova Blue cloning cells and confirmed by Sanger sequencing.

\section{Construction of TAB1 S395A and CAMKIV S189A variants}

Using QuickChange site-directed mutagenesis, single point mutations mutants were made in the pET28a-TAB1 or pET28a-CAMKIV parent plasmids by replacing the existing amino acid with the GCA (alanine) codon. PCR primers were designed to have $\sim 15-20$ base pairs of homology on either side of the mutation site. Each set of primers for a given variant were used to amplify the glycosite mutation using the parent plasmid as the template. The amplified PCR products were digested with the Dpn1 restriction enzyme to digest the parent plasmid, leaving the desired mutant plasmids. The digested DNA products were transformed into STELLAR or Nova Blue cloning cells and confirmed by Sanger sequencing.

\section{Bacterial expression and purification of 8XHIS-HRV3C-ncOGT variants}

Expression of 8XHIS-HRV3C-ncOGT WT, TPR truncation mutants, and TPR point mutants were carried out in LOBSTR (BL21-DE3) cells (Kerafast). $50 \mathrm{~mL}$ starter cultures of $50 \mu \mathrm{g} / \mathrm{mL}$ kanamycin supplemented LB media were grown at $37{ }^{\circ} \mathrm{C}(250 \mathrm{rpm})$ from a single colony before addition to $1.5 \mathrm{~L}$ of LB media supplemented with 50 $\mu \mathrm{g} / \mathrm{mL}$ kanamycin. Cells were grown at $37^{\circ} \mathrm{C}(250 \mathrm{rpm})$ to an OD600 of 1.0 . The cells were cooled to $16^{\circ} \mathrm{C}$ for 30 minutes, and expression was induced with $0.2 \mathrm{mM}$ isopropyl $\beta$-D-1-thiogalactopyranoside (IPTG) overnight at $16{ }^{\circ} \mathrm{C}$. The cells were pelleted by centrifugation at $4200 \mathrm{rpm}$ for 20 minutes and stored at $-80{ }^{\circ} \mathrm{C}$ until lysis.

For protein purifications, the cell pellets lysed via cell disruption and purified using Ni-NTA agarose superflow resin (Qiagen) as previously described. ${ }^{9}$ The eluates were supplemented with $1 \mathrm{mM}$ trishydroxypropylphosphine (THP) and concentrated using 100K MWCO Amicon concentration tubes (Millapore) for full-length ncOGT variants and $50 \mathrm{~K}$ MWCO Amicon concentration tubes for the ncOGT TPR truncation variants. The proteins were isolated through size-exclusion chromatography using a Superdex 200 increase 10/300 GL column (GE Life Sciences) with $1 \mathrm{X}$ TBS, $\mathrm{pH}$ 7.4. The resulting fractions' identity were verified by SDS-PAGE with appropriate molecular weight standards. Fractions containing the ncOGT variants were pooled and concentrated using the 100K MWCO Amicon concentration tube (50K MWCO tubes for TPR truncations). The concentrated protein was aliquoted and stored at $-80{ }^{\circ} \mathrm{C}$ until use. Before use, OGT aliquots were thawed on ice, and centrifuged at max speed for 20 minutes at $4{ }^{\circ} \mathrm{C}$ to pellet any aggregated protein. The supernatant was removed and store on ice until use, with the concentration measured using $A 280$ with the extinction coefficient of $117580 \mathrm{M}^{-1} \mathrm{~cm}^{-1}$ for fulllength ncOGT, $77240 \mathrm{M}^{-1} \mathrm{~cm}^{-1}$ for OGT 4.5, $80220 \mathrm{M}^{-1} \mathrm{~cm}^{-1}$ for OGT 5.5, $84690 \mathrm{M}^{-1} \mathrm{~cm}^{-1}$ for OGT 6.5, $82170 \mathrm{M}^{-}$ ${ }^{1} \mathrm{~cm}^{-1}$ for OGT 7.5, $93170 \mathrm{M}^{-1} \mathrm{~cm}^{-1}$ for OGT 8.0, and $100160 \mathrm{M}^{-1} \mathrm{~cm}^{-1}$ for OGT 9.0.

\section{In vitro glycosylation of HeLa extracts by ncOGT variants}

Protein glycosylation profiles for each ncOGT variants were determined in HeLa whole cell extracts that were prepared as previously described. ${ }^{8}$ For these experiments, HeLa cell extracts were thawed on ice and centrifuged at $4{ }^{\circ} \mathrm{C}$ for 20 minutes at $45,000 \mathrm{rpm}$ (Beckman Coulter TLA120.2 rotor) to pellet any remaining precipitated debris. Following centrifugation, the HeLa cell extracts were aliquoted to a final volume of $40 \mu \mathrm{L}$. The aliquots were incubated with $1 \mathrm{mM}$ of UDP-GIcNAc and equal volumes of reaction buffer $(20 \mathrm{mM}$ Tris, $\mathrm{pH}$ 7.4, $150 \mathrm{mM} \mathrm{NaCl}, 20 \mathrm{mM} \mathrm{MgCl} 2,1 \mathrm{mM}$ THP) and $1 \mu \mathrm{M}$ OGT for ncOGT D2A and D4A mutants or $2.5 \mu \mathrm{M}$ OGT for ncOGT N5A mutants at $37^{\circ} \mathrm{C}$. At 1 minute, 5 minutes, and 15 minutes, $10 \mu \mathrm{L}$ aliquots were taken from the reaction and quenched with $10 \mu \mathrm{L} 2 \mathrm{X}$ Laemmli loading buffer containing $\beta$-mercaptoethanol. The samples were boiled at $95{ }^{\circ} \mathrm{C}$ for 10 minutes and run on a 4-20\% TGX SDS-PAGE gel (Bio-Rad) at $180 \mathrm{~V}$ for 1.5 hours. The gels were transferred to PVDF membranes (BioRad) and analyzed by western blot with the anti-O-GIcNAc (CTD110.6, Cell Signaling), anti-OGT (D1D8Q, Cell Signaling), and anti-GAPDH (9484, Abcam) antibodies. Activity (densitometry) calculated in ImageJ by measuring the entire lane for each mutant, normalizing to the corresponding WT lane, and using the following equation: $\frac{\left[\left(\frac{m u t 1 \min }{w t 1 \min }\right)+\left(\frac{m u t 5 \min }{w t 5 \min }\right)+\left(\frac{m u t 15 \min }{w t 15 \min }\right)\right]}{3}$. Activity reported is an average of two biological replicates. 


\section{Bacterial expression and purification of OGT substrates}

Expression of pET28a-CARM1, CAMKIV, and TAB1 were carried out in LOBSTR (BL21-DE3) cells (Kerafast). $50 \mathrm{~mL}$ starter cultures of $50 \mu \mathrm{g} / \mathrm{mL}$ kanamycin supplemented LB media were grown at $37{ }^{\circ} \mathrm{C}(250 \mathrm{rpm})$ from a single colony before addition to $1.5 \mathrm{~L}$ of LB media supplemented with $50 \mu \mathrm{g} / \mathrm{mL}$ kanamycin. Cells were grown at $37{ }^{\circ} \mathrm{C}(250 \mathrm{rpm})$ to an OD600 of 0.6 . The cells were cooled to $16{ }^{\circ} \mathrm{C}$ for 30 minutes, and expression was induced with $0.2 \mathrm{mM}$ isopropyl $\beta$-D-1-thiogalactopyranoside (IPTG) overnight at $16{ }^{\circ} \mathrm{C}$. The cells were pelleted by centrifugation at $4200 \mathrm{rpm}$ for 20 minutes and stored at $-80{ }^{\circ} \mathrm{C}$ until lysis.

The cell pellets were resuspended in 1X TBS, pH 7.4 (50 mM Tris, pH 7.4, $150 \mathrm{mM} \mathrm{NaCl,} \mathrm{supplemented} \mathrm{with}$ $0.1 \mathrm{mg} / \mathrm{mL}$ lysozyme, $0.1 \mathrm{mg} / \mathrm{mL}$ DNAse I, and $1 \mathrm{mM} \mathrm{PMSF}$ ) at $4{ }^{\circ} \mathrm{C}$ for 30 minutes. The cells were lysed using an Avestin Emulsiflex C3 cell disruptor (ATA Scientific) three times at 15,000 psi and the debris was pelleted through centrifugation at $14,000 \mathrm{xg}$ for 20 minutes at $4{ }^{\circ} \mathrm{C}$. The supernatant was collected, and $40 \mathrm{mM}$ imidazole was added before the lysate was incubated with Ni-NTA agarose superflow resin (Qiagen) for 1 hour at $4{ }^{\circ} \mathrm{C}$, which was prewashed with 3 column volumes $1 \mathrm{X}$ TBS, $\mathrm{pH} 7.4+40 \mathrm{mM}$ imidazole for nickel affinity purification. The flow through was removed and the resin was washed with 10 column volumes of $1 \mathrm{X}$ TBS, $\mathrm{pH} 7.4+50 \mathrm{mM}$ imidazole. The protein was then eluted with 4 column volumes $1 \mathrm{X}$ TBS, pH $7.4+250 \mathrm{mM}$ imidazole. The eluate supplemented with $1 \mathrm{mM}$ THP and concentrated using a 30K MWCO Amicon concentration tube (Millapore). The protein was isolated through size-exclusion chromatography using a Superdex 75 increase 10/300 GL column (GE Life Sciences) with 1X TBS, pH 7.4. The resulting fractions' identity were verified by SDS-PAGE with appropriate molecular weight standards. Fractions contains the ncOGT variants were pooled and concentrated using the $30 \mathrm{~K}$ MWCO Amicon concentration tube. The concentrated protein was aliquoted and stored at $-80{ }^{\circ} \mathrm{C}$ until use. Before use, OGT aliquots were thawed on ice, and centrifuged at max speed for 20 minutes at $4{ }^{\circ} \mathrm{C}$ to pellet any aggregated protein. The supernatant was removed and store on ice until use, with the concentration measured using A280 with the extinction coefficient of $61170 \mathrm{M}^{-1} \mathrm{~cm}^{-1}$ for CARM1, $48360 \mathrm{M}^{-}$ ${ }^{1} \mathrm{~cm}^{-1}$ for CAMKIV, and $37360 \mathrm{M}^{-1} \mathrm{~cm}^{-1}$ for TAB1.

\section{In vitro glycosylation of OGT substrates by ncOGT variants}

ncOGT variants and the purified substrates, CARM1, CAMKIV, and TAB1 were thawed on ice, and centrifuged at $4{ }^{\circ} \mathrm{C}$ for 20 minutes at max speed (Eppendorf 5424R) to pellet any remaining precipitated debris. Following centrifugation, $2.5 \mu \mathrm{M}$ OGT, $15 \mu \mathrm{M}$ substrate, $1 \mathrm{mM}$ UDP-GlcNAc were incubated in reaction buffer (20 mM Tris, $\mathrm{pH} 7.4,150 \mathrm{mM} \mathrm{NaCl}, 20 \mathrm{mM} \mathrm{MgCl} 2,1 \mathrm{mM}$ THP) at a final volume of $20 \mu \mathrm{L}$ for 90 minutes at $37^{\circ} \mathrm{C}, 350 \mathrm{rpm}$. The reactions were quenched with $20 \mu \mathrm{L} 2 \mathrm{X}$ Laemmli loading buffer containing $\beta$-mercaptoethanol. The samples were boiled at $95{ }^{\circ} \mathrm{C}$ for 10 minutes and run on a 4-20\% TGX SDS-PAGE gel (Bio-Rad) at $180 \mathrm{~V}$ for 1.5 hours. The gels were transferred to nitrocellulose membranes and analyzed by western blot with the anti-O-GlcNAc (CTD110.6, Cell Signaling) primary antibody and anti-mouse IgG-680RD IRDye (LI-COR) secondary antibody. Activity (densitometry) reported in Tables S3-5 calculated in ImageJ by measuring the densitometry of substrate glycosylation band for each mutant, normalizing to the corresponding WT lane using the following equation: $\frac{\text { Mutant activity }}{\text { WT activity }}$. Activity reported is an average of two replicates.

\section{In vitro turnover assay of TAB1 glycosylation by ncOGT variants}

Turnover rates of TAB1 WT and S395A glycosylation for ncOGT WT and D2A-2 prox were determined using an in vitro glycosylation time course followed by western blotting. For each reaction, $1 \mu \mathrm{M}$ ncOGT variant, $20 \mu \mathrm{M}$ TAB1, and $1 \mathrm{mM}$ UDP-GlcNAc were incubated in reaction buffer $(20 \mathrm{mM}$ Tris, pH 7.4, $150 \mathrm{mM} \mathrm{NaCl}, 20 \mathrm{mM}$ $\mathrm{MgCl}_{2}, 1 \mathrm{mM}$ THP) at a total volume of $90 \mu \mathrm{L}$. Reactions were incubated at $37^{\circ} \mathrm{C}$ with $350 \mathrm{rpm}$ shaking. At 0,1 , $2.5,5,10,15,20$, and 30 minutes for TAB1 WT and 0, 1, 5, 10, 15, 20, and 30 minutes for TAB1 S395A, $10 \mu \mathrm{L}$ of each reaction was removed and quenched with $10 \mu \mathrm{L} 2 \mathrm{X}$ Laemmli loading buffer containing $\beta$ mercaptoethanol. The samples were boiled at $95^{\circ} \mathrm{C}$ for 10 minutes. The samples were split between two 4-20\% TGX SDS-PAGE gels (Bio-Rad) and ran at $180 \mathrm{~V}$ for 1.5 hours. One gel was stained with Pierce Imperial ${ }^{\mathrm{TM}}$ protein stain according to the manufacturer's protocol, while the other was transferred to a nitrocellulose membrane and analyzed by western blot with the anti-O-GIcNAc (CTD110.6, Cell Signaling) primary antibody and anti-mouse IgG-680RD IRDye (LI-COR) secondary antibody. Activity (densitometry) reported in Figure 4G and Tables S6-7 was calculated in ImageJ for each timepoint by measuring the densitometry of the full-length glycosylated substrate band. Substrate turnover by ncOGT variants reported in Figure 4G was calculated by fitting the linear ranges of the activity curves and taking the slopes of the resultant lines of best fit. Relative 
turnover was calculated using the following equation: $\frac{\text { slope of mutant turnover }}{\text { slope of } W T \text { turnover }}$. Data reported is an average of two replicates.

\section{In vitro time-dependent glycosylation of CAMKIV by ncOGT variants}

The time dependent of CAMKIV WT and CAMKIV S189A glycosylation by ncOGT WT and D2A-3med were determined using an in vitro glycosylation time course followed by western blotting. For each reaction, $2.5 \mu \mathrm{M}$ ncOGT variant, $15 \mu \mathrm{M}$ CAMKIV variant, and $1 \mathrm{mM}$ UDP-GlcNAc were incubated in reaction buffer $(20 \mathrm{mM}$ Tris, $\mathrm{pH} 7.4,150 \mathrm{mM} \mathrm{NaCl}, 20 \mathrm{mM} \mathrm{MgCl}, 1 \mathrm{mM} \mathrm{THP}$ ) at a total volume of $55 \mu \mathrm{L}$. Reactions were incubated at $37^{\circ} \mathrm{C}$ with 350 rpm shaking. At 0, 15, 30, 60, and 90 minutes for CAMKIV WT and 0, 5, 15, 30, and 60 minutes for CAMKIV S189A, $10 \mu \mathrm{L}$ of each reaction was removed and quenched with $10 \mu \mathrm{L} 2 \mathrm{X}$ Laemmli loading buffer containing $\beta$-mercaptoethanol. The samples were boiled at $95^{\circ} \mathrm{C}$ for 10 minutes. The samples were split between two $4-20 \%$ TGX SDS-PAGE gels (Bio-Rad) and ran at $180 \mathrm{~V}$ for 1.5 hours. One gel was stained using InstantBlue ${ }^{\mathrm{TM}}$ protein stain (Sigma-Aldrich) according to the manufacturer's protocol, while the other was transferred to a nitrocellulose membrane and analyzed by western blot using the anti-O-GIcNAc (CTD110.6, Cell Signaling) primary antibody and anti-mouse IgG-680RD IRDye (LI-COR) secondary antibody.

\section{References}

1. Jinek, M.; Rehwinkel, J.; Lazarus, B.D.; Izaurralde, E.; Hanover, J.A.; Conti, E., Nature Structural \& Molecular Biology 2004, 11(10), 1001.

2. Ashkenazy, H.; Abadi, S.; Martz, E.; Chay, O.; Mayrose, I.; Pupko, T.; Ben-Tal, N., Nucleic Acids Research 2016, 44, W344.

3. Ahskenazy, H.; Erez, E.; Martz, E.; Pupko, T.; Ben-Tal, N. Nucleic Acids Research 2010, 38, W529.

4. Shen, D.L.; Gloster, T.M.; Yuzwa, S.A.; Vocadlo, D.J. Journal of Biological Chemistry 2012, 287(19), 15395.

5. Lazarus, M.B.; Nam, Y.; Jiang, J.; Sliz, P.; Walker, S. Nature 2011, 469(7331), 564.

6. Gross, B.J.; Kraybill, B.C., Walker, S. J. Am. Chem. Soc. 2005, 127(42), 14588.

7. Lazarus, M.B.; Jiang, J.; Kapuria, V.; Bhuiyan, T.; Janetzko, J.; Zandberg, W.F.; Vocadlo, D.J.; Walker, S. Science 2013, 342(6163), 1235.

8. Levine, Z.G.; Fan, C.; Melicher, M.S.; Orman, M.; Benjamin, T.; Walker, S. J. Am. Chem. Soc. 2018, 140(10), 3510.

9. Joiner, C.M.; Levine, Z.G.; Aonbangkhen, C.; Woo, C.M.; Walker, S. J. Am. Chem. Soc. 2019, 141(33), 12974. 